\title{
Formalization of Human Categorization Process Using Interpolative Boolean Algebra
}

\author{
Vladimir Dobrić, ${ }^{1}$ Darko Kovačević, ${ }^{1}$ Bratislav Petrović, ${ }^{1}$ \\ Dragan Radojević, ${ }^{2}$ and Pavle Milošević ${ }^{1}$ \\ ${ }^{1}$ Faculty of Organizational Sciences, University of Belgrade, Jove Ilića 154, 11000 Belgrade, Serbia \\ ${ }^{2}$ Institute Mihajlo Pupin, University of Belgrade, Volgina 15, 11000 Belgrade, Serbia
}

Correspondence should be addressed to Vladimir Dobrić; vdobric@gmail.com

Received 1 August 2014; Accepted 21 September 2014

Academic Editor: Shifei Ding

Copyright (C) 2015 Vladimir Dobrić et al. This is an open access article distributed under the Creative Commons Attribution License, which permits unrestricted use, distribution, and reproduction in any medium, provided the original work is properly cited.

\begin{abstract}
Since the ancient times, it has been assumed that categorization has the basic form of classical sets. This implies that the categorization process rests on the Boolean laws. In the second half of the twentieth century, the classical theory has been challenged in cognitive science. According to the prototype theory, objects belong to categories with intensities, while humans categorize objects by comparing them to prototypes of relevant categories. Such categorization process is governed by the principles of perceived world structure and cognitive economy. Approaching the prototype theory by using truth-functional fuzzy logic has been harshly criticized due to not satisfying the complementation laws. In this paper, the prototype theory is approached by using structure-functional fuzzy logic, the interpolative Boolean algebra. The proposed formalism is within the Boolean frame. Categories are represented as fuzzy sets of objects, while comparisons between objects and prototypes are formalized by using Boolean consistent fuzzy relations. Such relations are directly constructed from a Boolean consistent fuzzy partial order relation, which is treated by Boolean implication. The introduced formalism secures the principles of categorization showing that Boolean laws are fundamental in the categorization process. For illustration purposes, the artificial cognitive system which mimics human categorization activity is proposed.
\end{abstract}

\section{Introduction}

Categorization is the process in which ideas and objects are recognized, differentiated, and understood [1]. Humans and other organisms consider objects and events as members of categories. Such cognitive activity is automatic and effortless. Categories structure our knowledge about the world. The ability to categorize is fundamental to any natural or artificial cognitive system. Since the time of ancient Greece, it has been assumed that an object can belong or cannot belong to a category and that humans categorize by identifying necessary and sufficient conditions for an object to belong to the category. All objects satisfying such conditions are equivalent with respect to that category. This can be formalized by using classical set theory. The meanings of the categories can be explained by the operations of classical logic. Such formalization implies that the categorization process is governed by the laws of Boolean algebra, among which the laws of thought are fundamental. This view on the categorization is called the classical view [2-4].

In the second half of the twentieth century, the classical theory has been challenged in cognitive science. From this point of view, the categorization is based on gradation. This means that objects can belong to categories with intensities. Two fundamental principles of categorization have been proposed in [5]. According to the principle of perceived world structure, the perceived world comes as structured information. In accordance with the principle of cognitive economy, the task of the categorization is to provide maximum information about the world with the least cognitive effort. This is possible thanks to gradation. In the classical case, two objects can be differentiated if one belongs, while the other one does not belong, to the relevant category. When more objects have to be differentiated from each other, 
more categories have to be considered in the process of cognition, which requires more cognitive effort. In the case of gradation, an infinite number of objects can be differentiated inside one category. This implies that the categorization based on gradation can provide maximum information about the world with the least cognitive effort.

According to the gradation (prototype) theory, humans categorize objects by comparing them to prototypes of relevant categories [5-7]. As in [8], we use the term prototype in the most general sense. Under this term, we assume either an abstract summary of a category, or a set of exemplars-actual category members pulled from memory. Since the classical theory has been approached by using classical logic/set theory, the prototype theory should be approached by using fuzzy logic/set theory. In the conventional fuzzy logic [9], the laws of complements are not taken as axioms. This has been harshly criticized in cognitive science [10], as such laws have been considered fundamental from the time of Aristotle. According to [11], the laws of complements cannot be satisfied in the conventional fuzzy logic because it follows the principle of truth functionality. According to this principle, the value of any logical expression can be calculated by using the values of its components. The principle is valid in classical Boolean algebra, but it breaks the Boolean frame in the case of gradation. Fuzzy logic, which secures the laws of thought and the Boolean frame, has been proposed in [12]. In opposition to conventional fuzzy logics, this approach is based on the fundamental principle of structure functionality.

In this paper, the Boolean consistent approach to gradation-interpolative Boolean algebra-is used in an attempt at the formalization of human categorization activity. It is assumed that this innate human activity follows the prototype theory. The proposed formalism is within the Boolean frame, securing the principles of categorization. This shows that Boolean laws are universal in categorizationvalid in the classical and the prototype theory. Such laws can be considered as the fundamental laws governing the categorization process. For illustration purposes, the simple artificial cognitive system which mimics human categorization ability is proposed.

\section{Interpolative Boolean Algebra}

Interpolative Boolean algebra is the Boolean consistent fuzzy logic $[11,12]$. From the mathematical point of view, fuzzy logic rests on the principle of incompatibility [13]. According to this principle, increase in problem complexity diminishes our ability to solve the problem by using classical mathematical approaches (based on classical logic/set theory). According to [14], fuzzy logic brings a drastic reduction of complexity immanent to classical approaches when dealing with realworld problems, and fuzzy approaches offer more expressive power with less complexity in comparison to the classical ones. From the cognitive science point of view, fuzzy logic is an attempt at the formalization of remarkable human capabilities [15]. According to [15-17], fuzzy logic is inspired by the brain's crucial ability to manipulate perceptions and is essential for complex problems in artificial intelligence, such as the pattern recognition problem. This is the problem approached in this paper.

Fuzzy logic has been challenged in mathematics and cognitive science since its introduction. The main drawback of conventional fuzzy logics, based on the truth functionality principle, is the fact that they are not in the Boolean frame [14]. From the point of view of mathematics, this implies that the classical results cannot be directly generalized. For example, a basic concept of preference modeling is a preference structure. In the classical case, a preference structure can be constructed from a reflexive preference relation. In the case of gradation, this result is not possible in conventional fuzzy logics [18]. In cognitive science, fuzzy logic has been harshly criticized due to not satisfying the laws of complements [19-21]. Such laws have been considered as the fundamental laws of rational thinking since the ancient times. The laws of thought must apply without exception to any subject matter of thought. Boolean laws cannot be secured in conventional fuzzy logics as they are based on the truth functionality principle. In contrast to these fuzzy logics, interpolative Boolean algebra is based on the principle of structure functionality $[12,22]$. According to this principle, logical expressions have vector nature. The truth functionality is valid in the classical case as attention is reduced to only one vector component. In the fuzzy case, all vector components have to be used for Boolean consistent calculations. The structure vector of any logical expression can be calculated by using the structures of expression's components. The structure functionality is the fundamental Boolean principle as it secures the Boolean frame in any value realization of the algebra (binary, multivalued, or fuzzy).

Formally, the finite or atomic Boolean algebra $\mathrm{BA}(\Omega)=$ $P(P(\Omega))$ is generated by a set of free variables $\Omega=$ $\left\{x_{1}, x_{2}, \ldots, x_{m}\right\}$. Set $P(\Omega)$ is the power set of $\Omega$. For any $x_{i}, x_{j}, x_{k} \in \Omega$, Boolean laws are given by the following expressions.

Associativity:

$$
\begin{aligned}
& x_{i} \vee\left(x_{j} \vee x_{k}\right)=\left(x_{i} \vee x_{j}\right) \vee x_{k} \\
& x_{i} \wedge\left(x_{j} \wedge x_{k}\right)=\left(x_{i} \wedge x_{j}\right) \wedge x_{k} .
\end{aligned}
$$

Commutativity:

$$
\begin{aligned}
& x_{i} \vee x_{j}=x_{j} \vee x_{i}, \\
& x_{i} \wedge x_{j}=x_{j} \wedge x_{i} .
\end{aligned}
$$

Distributivity:

$$
\begin{aligned}
& x_{i} \wedge\left(x_{j} \vee x_{k}\right)=\left(x_{i} \wedge x_{j}\right) \vee\left(x_{i} \wedge x_{k}\right) \\
& x_{i} \vee\left(x_{j} \wedge x_{k}\right)=\left(x_{i} \vee x_{j}\right) \wedge\left(x_{i} \vee x_{k}\right)
\end{aligned}
$$

Identity:

$$
\begin{array}{ll}
x_{i} \vee \underline{0}=x_{i}, & x_{i} \wedge \overline{1}=x_{i} \\
x_{i} \wedge \underline{0}=\underline{0}, & x_{i} \vee \overline{1}=\overline{1}
\end{array}
$$


Idempotence:

$$
x_{i} \vee x_{i}=x_{i}, \quad x_{i} \wedge x_{i}=x_{i} .
$$

Absorption:

$$
\begin{aligned}
& x_{i} \wedge\left(x_{i} \vee x_{j}\right)=x_{i}, \\
& x_{i} \vee\left(x_{i} \wedge x_{j}\right)=x_{i} .
\end{aligned}
$$

\section{Complementation:}

$$
x_{i} \wedge \neg x_{i}=\underline{0}, \quad x_{i} \vee \neg x_{i}=\overline{1} .
$$

De Morgan laws:

$$
\begin{aligned}
& \neg\left(x_{i} \wedge x_{j}\right)=\neg x_{i} \vee \neg x_{j}, \\
& \neg\left(x_{i} \vee x_{j}\right)=\neg x_{i} \wedge \neg x_{j} .
\end{aligned}
$$

Here $\underline{0}$ and $\overline{1}$ are the smallest and the biggest elements of $\operatorname{BA}(\Omega)$.

Atomic elements $\alpha_{S}\left(x_{1}, x_{2}, \ldots, x_{m}\right)$ of $\mathrm{BA}(\Omega)$ are defined by the following expression:

$$
\alpha_{S}\left(x_{1}, x_{2}, \ldots, x_{m}\right)=\wedge_{x_{i} \in S} x_{i} \underset{x_{j} \in \Omega \backslash S}{\wedge} \neg x_{j} .
$$

Here $S \in P(\Omega)$.

Atomic elements have the following properties:

$$
\begin{gathered}
\alpha_{i}\left(x_{1}, x_{2}, \ldots, x_{m}\right) \cap \alpha_{j}\left(x_{1}, x_{2}, \ldots, x_{m}\right) \\
= \begin{cases}\underline{0}, & i \neq j \\
\alpha_{i}\left(x_{1}, x_{2}, \ldots, x_{m}\right), & i=j\end{cases} \\
\bigcup_{S \in P(\Omega)} \alpha_{S}\left(x_{1}, x_{2}, \ldots, x_{m}\right)=\overline{1} .
\end{gathered}
$$

Any element of Boolean algebra can be defined as union of relevant atoms. Which atoms are relevant for (included in) $\varphi\left(x_{1}, x_{2}, \ldots, x_{m}\right) \in \mathrm{BA}(\Omega)$ is defined by element's structure function $\sigma_{\varphi}\left(\alpha_{S}\right)$. Structure function is defined by the following expression:

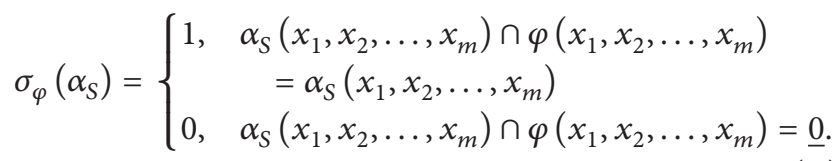

Here $S \in P(\Omega)$.

Any element $\varphi\left(x_{1}, x_{2}, \ldots, x_{m}\right) \in \mathrm{BA}(\Omega)$ can be defined by the following expression:

$$
\varphi\left(x_{1}, x_{2}, \ldots, x_{m}\right)=\bigcup_{S \in P(\Omega) \mid \sigma_{\varphi}\left(\alpha_{S}\right)=1} \alpha_{S}\left(x_{1}, x_{2}, \ldots, x_{m}\right) .
$$

Information on which atoms are relevant for $\varphi\left(x_{1}, x_{2}\right.$, $\left.\ldots, x_{m}\right) \in \mathrm{BA}(\Omega)$ is stored in structure vector defined by the following expression:

$$
\vec{\sigma}_{\varphi}=\left[\sigma_{\varphi}\left(\alpha_{S}\right) \mid S \in P(\Omega)\right]^{T}
$$

The structure of any combined element of $\mathrm{BA}(\Omega)$ can be directly calculated from the structures of its component:

$$
\begin{gathered}
\vec{\sigma}_{\phi \wedge \psi}=\vec{\sigma}_{\phi} \wedge \vec{\sigma}_{\psi}, \\
\vec{\sigma}_{\phi \vee \psi}=\vec{\sigma}_{\phi} \vee \vec{\sigma}_{\psi}, \\
\vec{\sigma}_{\neg \phi}=\neg \vec{\sigma}_{\phi}=\overrightarrow{1}-\vec{\sigma}_{\phi} .
\end{gathered}
$$

The value $v\left(\varphi\left(x_{1}, x_{2}, \ldots, x_{m}\right)\right) \in[0,1]$ of any $\varphi\left(x_{1}, x_{2}\right.$, $\left.\ldots, x_{m}\right) \in \operatorname{BA}(\Omega)$ is given by corresponding Boolean polynomial, which is the sum of values of relevant atomic elements:

$$
v\left(\varphi\left(x_{1}, x_{2}, \ldots, x_{m}\right)\right)=\sum_{S \in P(\Omega) \mid \sigma_{\varphi}(S)=1} v\left(\alpha_{S}\left(x_{1}, x_{2}, \ldots, x_{m}\right)\right) .
$$

The value $v\left(\alpha_{S}\left(x_{1}, x_{2}, \ldots, x_{m}\right)\right) \in[0,1]$ of atomic element $\alpha_{S}\left(x_{1}, x_{2}, \ldots, x_{m}\right) \in \mathrm{BA}(\Omega)$ is given by corresponding atomic Boolean polynomial:

$$
v\left(\alpha_{S}\left(x_{1}, x_{2}, \ldots, x_{m}\right)\right)=\underset{x_{i} \in S}{\otimes} v\left(x_{i}\right) \underset{x_{j} \in \Omega \backslash S}{\otimes}\left(1-v\left(x_{j}\right)\right) .
$$

Here $v\left(x_{i}\right) \in[0,1]$ is the fuzzy value of $x_{i} \in \Omega, S \in P(\Omega)$ and $\otimes:[0,1] \times[0,1] \rightarrow[0,1]$ is a generalized product operator which can be any $T$-norm.

For example, for $\Omega=\left\{x_{i}, x_{j}\right\}$ the generalized product operator has the following property:

$$
\begin{aligned}
\max \left(v\left(x_{i}\right)+v\left(x_{j}\right)-1,0\right) & \leq v\left(x_{i}\right) \otimes v\left(x_{j}\right) \\
& \leq \min \left(v\left(x_{i}\right), v\left(x_{j}\right)\right) .
\end{aligned}
$$

The value of any $\varphi\left(x_{1}, x_{2}, \ldots, x_{m}\right) \in \mathrm{BA}(\Omega)$ can be represented as the scalar product of two vectors:

$$
v\left(\varphi\left(x_{1}, x_{2}, \ldots, x_{m}\right)\right)=\vec{\sigma}_{\varphi} \vec{\alpha}
$$

Here $\vec{\sigma}_{\varphi}$ is the structure vector of $\varphi\left(x_{1}, x_{2}, \ldots, x_{m}\right) \in \mathrm{BA}(\Omega)$ and $\vec{\alpha}$ is the vector of atomic Boolean polynomials.

Vector of atomic Boolean polynomials $\vec{\alpha}$ is defined by the following expression:

$$
\vec{\alpha}=\left[v\left(\alpha_{S}\left(x_{1}, x_{2}, \ldots, x_{m}\right)\right) \mid S \in P(\Omega)\right]^{T} .
$$


According to the principle of structure functionality, Boolean consistent fuzzy value of any element of $\mathrm{BA}(\Omega)$ can be calculated by the following rules [22]:

$$
\begin{array}{r}
v\left(\varphi_{i}\left(x_{1}, \ldots, x_{m}\right) \wedge \varphi_{j}\left(x_{1}, \ldots, x_{m}\right)\right) \\
={ }_{\mathrm{def}} v\left(\varphi_{i}\left(x_{1}, \ldots, x_{m}\right)\right) \otimes v\left(\varphi_{j}\left(x_{1}, \ldots, x_{m}\right)\right), \\
v\left(\varphi_{i}\left(x_{1}, \ldots, x_{m}\right) \vee \varphi_{j}\left(x_{1}, \ldots, x_{m}\right)\right) \\
={ }_{\mathrm{def}} v\left(\varphi_{i}\left(x_{1}, \ldots, x_{m}\right)\right)+v\left(\varphi_{j}\left(x_{1}, \ldots, x_{m}\right)\right) \\
\quad-v\left(\varphi_{i}\left(x_{1}, \ldots, x_{m}\right)\right) \otimes v\left(\varphi_{j}\left(x_{1}, \ldots, x_{m}\right)\right), \\
v\left(\neg \varphi_{i}\left(x_{1}, \ldots, x_{m}\right)\right)={ }_{\mathrm{def}} 1-v\left(\varphi_{i}\left(x_{1}, \ldots, x_{m}\right)\right), \\
v\left(x_{i} \wedge x_{j}\right)={ }_{\mathrm{def}}\left\{\begin{array}{l}
v\left(x_{i}\right) \otimes v\left(x_{j}\right) \quad i \neq j, \\
v\left(x_{i}\right),
\end{array}\right. \\
v\left(x_{i} \vee x_{j}\right)={ }_{\mathrm{def}} v\left(x_{i}\right)+v\left(x_{j}\right)-v\left(x_{i}\right) \otimes v\left(x_{j}\right), \\
v\left(\neg x_{i}\right)={ }_{\mathrm{def}} 1-v\left(x_{i}\right) .
\end{array}
$$

Here $\varphi_{i}\left(x_{1}, \ldots, x_{m}\right), \varphi_{j}\left(x_{1}, \ldots, x_{m}\right) \in \operatorname{BA}(\Omega), v\left(\varphi_{i}\left(x_{1}\right.\right.$, $\left.\left.\ldots, x_{m}\right)\right), v\left(\varphi_{j}\left(x_{1}, \ldots, x_{m}\right)\right) \in[0,1], x_{1}, \ldots, x_{m} \in \Omega$, $v\left(x_{1}\right), \ldots, v\left(x_{m}\right) \in[0,1]$.

Software tool for calculations of Boolean consistent fuzzy values of elements of $\mathrm{BA}(\Omega)$ is proposed in [23].

Generalized product $\otimes:[0,1] \times[0,1] \rightarrow[0,1]$ can be any operator that satisfies the following properties.

Commutativity:

$$
v\left(x_{i}\right) \otimes v\left(x_{j}\right)=v\left(x_{i}\right) \otimes v\left(x_{j}\right) .
$$

Associativity:

$$
\left(v\left(x_{i}\right) \otimes v\left(x_{j}\right)\right) \otimes v\left(x_{k}\right)=v\left(x_{i}\right) \otimes\left(v\left(x_{j}\right) \otimes v\left(x_{k}\right)\right) .
$$

Monotonicity:

$$
v\left(x_{i}\right) \leq v\left(x_{j}\right) \Longrightarrow v\left(x_{i}\right) \otimes v\left(x_{k}\right) \leq v\left(x_{j}\right) \otimes v\left(x_{k}\right) .
$$

Boundary:

$$
v\left(x_{i}\right) \otimes 1=v\left(x_{i}\right) .
$$

Nonnegativity:

$$
\underset{x_{i} \in S}{\otimes} v\left(x_{i}\right) \underset{x_{j} \in \Omega \backslash S}{\otimes}\left(1-v\left(x_{j}\right)\right) \geq 0 .
$$

Here $\Omega=\left\{x_{1}, x_{2}, \ldots, x_{m}\right\}, S \in P(\Omega)$.

In the following section, the prototype theory will be formalized by using interpolative Boolean algebra.

\section{Formalization of Human Categorization Process}

According to the prototype theory, humans categorize objects by comparing them to prototypes of relevant categories.
Prototypes or cognitive reference points are formed in the process of cognition by considering observed (actual) members of the categories. The prototype theory is based on the principle of perceived world structure and the principle of cognitive economy. According to these principles, categories with graded structure "spring" to mind whenever objects or events are considered in the process of cognition. This provides maximum information about the world with the least cognitive effort. It is assumed in this paper that the categorization rests on cognitive acts of ordering of perceived objects and prototypes of relevant categories. The ordering must be relevant to a cognitive task at hand, requiring the least cognitive effort. From the information provided by such ordering, maximum information about the world can be acquired in the process of cognition. This way, the principles of categorization are secured.

Formally, an object considered in the process of cognition will be represented by using vector $\left[A_{1}(x), \ldots, A_{n}(x)\right]$, where $A_{i}(x) \in[0,1], i=1, \ldots, n$, are the properties of object $x$. Set $X$ is the set of objects for categorization. Set $Y$ is the set of prototypes of relevant categories. The prototype of a category is an object or a set of objects, statistically derived or selected from the observed category members. For example, the prototype of category " $C$ " can be represented as an object $y_{C} \in Y$, whose properties are the averages of properties of all category members:

$$
\begin{aligned}
y_{C} & =\frac{1}{|C|} \sum_{z \in C} z=\left[\frac{1}{|C|} \sum_{z \in C} A_{1}(z), \ldots, \frac{1}{|C|} \sum_{z \in C} A_{n}(z)\right] \\
& =\left[A_{1}\left(y_{C}\right), \ldots, A_{n}\left(y_{C}\right)\right] .
\end{aligned}
$$

Here $y_{C} \in[0,1]^{n}$ is the prototype of category $C, z \in[0,1]^{n}$ is the member of $C$, and $|C|$ is the cardinality of $C$.

Alternatively, the prototype of a category can be defined as the set of the most representative objects of the category (according to some criteria), or as the set which contains all members of the category. Approach to categorization, according to which humans categorize objects by comparing them to actual category members, is called the exemplar approach $[24,25]$. The categorization based on exemplars is more flexible but less economical, in comparison to the first approach. Considering exemplars in the process of cognition secures better categorization of "atypical" objects but requires more cognitive effort.

The ordering of objects and prototypes will be formalized by using a Boolean consistent fuzzy partial order relation defined over $X \cup Y$. This reflexive, antisymmetric and transitive binary (two-place) fuzzy relation will be called the primitive relation and will be treated by using Boolean implication. The fuzzy partial order relation can be defined by the following expression:

$$
\begin{aligned}
& (x \leq y) \\
& =v\left(\phi_{i}\left(A_{1}(x), \ldots, A_{n}(x)\right) \Longrightarrow \phi_{i}\left(A_{1}(y), \ldots, A_{n}(y)\right)\right) \\
& =v\left(\neg \phi_{i}\left(A_{1}(x), \ldots, A_{n}(x)\right) \vee \phi_{i}\left(A_{1}(y), \ldots, A_{n}(y)\right)\right)
\end{aligned}
$$




$$
\begin{aligned}
& =1-v\left(\phi_{i}\left(A_{1}(x), \ldots, A_{n}(x)\right)\right) \\
& +\min \left(v\left(\phi_{i}\left(A_{1}(x), \ldots, A_{n}(x)\right)\right),\right. \\
& \left.v\left(\phi_{i}\left(A_{1}(y), \ldots, A_{n}(y)\right)\right)\right) .
\end{aligned}
$$

Here $(x \leq y) \in[0,1]$ is a fuzzy partial order relation (the proof is straightforward), $x, y \in[0,1]^{n}, x \in X$, $y \in Y, \phi_{i}\left(A_{1}(x), \ldots, A_{n}(x)\right)$ can be any Boolean function, $v\left(\phi_{i}\left(A_{1}(x), \ldots, A_{n}(x)\right)\right) \in[0,1]$ is the function's value, and $1-v\left(\phi_{i}\left(A_{1}(x), \ldots, A_{n}(x)\right)\right)+\min \left(v\left(\phi_{i}\left(A_{1}(x), \ldots, A_{n}(x)\right)\right)\right.$, $\left.v\left(\phi_{i}\left(A_{1}(y), \ldots, A_{n}(y)\right)\right)\right)$ is the Boolean polynomial which corresponds to Boolean implication $\phi_{i}\left(A_{1}(x), \ldots, A_{n}(x)\right) \Rightarrow$ $\phi_{i}\left(A_{1}(y), \ldots, A_{n}(y)\right)$.

Less complex primitive relations can be defined by the following expressions:

$$
\begin{aligned}
(x \leq y) & =\sum_{i=1, \ldots, n} w_{i} v\left(A_{i}(x) \Longrightarrow A_{i}(y)\right) \\
& =\sum_{i=1, \ldots, n} w_{i} v\left(\neg A_{i}(x) \vee A_{i}(y)\right) \\
& =\sum_{i=1, \ldots, n} w_{i}\left(1-A_{i}(x)+\left(\min A_{i}(x), A_{i}(y)\right)\right), \\
(x \leq y) & =v\left(\hat{i=1, \ldots, n}_{i}\left(A_{i}(x) \Longrightarrow A_{i}(y)\right)\right) \\
& =v\left(\sum_{i=1, \ldots, n}^{\wedge}\left(\neg A_{i}(x) \vee A_{i}(y)\right)\right) \\
& =\underset{i=1, \ldots, n}{\otimes} v\left(\neg A_{i}(x) \vee A_{i}(y)\right) \\
& =\underset{i=1, \ldots, n}{\otimes}\left(1-A_{i}(x)+\min \left(A_{i}(x), A_{i}(y)\right)\right) .
\end{aligned}
$$

Here $w_{i} \in[0,1], \sum_{i=1, \ldots, n} w_{i}=1$, the generalized product operator $(\otimes)$ can be any $t$-norm, and $v\left(A_{i}(x) \Rightarrow A_{i}(y)\right)=1-$ $A_{i}(x)+\min \left(A_{i}(x), A_{i}(y)\right)$ is the Boolean polynomial which corresponds (gives value) to Boolean implication $A_{i}(x) \Rightarrow$ $A_{i}(y)=\neg A_{i}(x) \vee A_{i}(y)$.

Pseudological expressions, such as the first expression in (28), are introduced in [26].

Comparisons between object $x \in X$ and prototype $y \in Y$ will be formalized on the basis of primitive relation $(x \leq$ $y) \in[0,1]$ and its inverse $(x \geq y) \in[0,1], x, y \in[0,1]^{n}$, $(x \geq y)=(x \leq y)^{-1}$. By using the information provided by these relations, Boolean algebra of fuzzy relations $\mathrm{BA}(\Omega)=$ $P(P(\Omega))$ can be generated, where $P(\Omega)$ is the power set of $\Omega=\{(x \leq y),(x \geq y)\}$. Elements of this algebra have semantics (meanings) thanks to the principle of structure functionality. Such elements formalize the cognitive acts of comparisons between objects and prototypes, on the basis of which the objects are categorized. Proposed formalization of prototype theory secures the principles of categorization. The cognitive effort (in this case computational effort) is minimized as the artificial process of categorization is only based on the primitive relation. At the same time, maximum information about the world can be directly derived from the information provided by the primitive relation. This implies that an artificial cognitive system based on the proposed formalism can acquire maximum information about the world with the least computational effort. In the rest of this section, elements of the Boolean algebra of fuzzy relations are defined. Detailed information on Boolean consistent fuzzy relations can be found in [27].

Atomic elements (atomic relations) of $\mathrm{BA}(\Omega)$ are the following.

Reflexive, symmetric and transitive fuzzy equivalence (or similarity) relation:

$$
(x=y)=(x \leq y) \wedge(x \geq y)=(x \leq y) \otimes(x \geq y) .
$$

Irreflexive, asymmetric and transitive fuzzy strict order relations:

$$
\begin{aligned}
(x>y) & =\neg(x \leq y) \wedge(x \geq y)=(1-(x \leq y)) \otimes(x \geq y) \\
& =(x \geq y)-(x \leq y) \otimes(x \geq y), \\
(x<y) & =(x \leq y) \wedge \neg(x \geq y)=(x \leq y) \otimes(1-(x \geq y)) \\
& =(x \leq y)-(x \leq y) \otimes(x \geq y) .
\end{aligned}
$$

Irreflexive, symmetric and transitive fuzzy incomparability relation:

$$
\begin{aligned}
(x<>y) & =\neg(x \leq y) \wedge \neg(x \geq y) \\
& =(1-(x \leq y)) \otimes(1-(x \geq y)) \\
& =1-(x \geq y)-(x \leq y)+(x \leq y) \otimes(x \geq y) .
\end{aligned}
$$

In these equations, operator of generalized product $\otimes$ can be any $T$-norm. According to the structure functionality principle, other Boolean consistent fuzzy relations/elements of $\mathrm{BA}(\Omega)$ can be defined as unions (sums) of relevant atomic relations. Fuzzy partial order relations are defined by the following expressions:

$$
\begin{aligned}
(x \leq y) & =(x<y) \vee(x=y)=(x<y)+(x=y) \\
& =(x \leq y)-(x \leq y) \otimes(x \geq y)+(x \leq y) \otimes(x \geq y) \\
(x \geq y) & =(x>y) \vee(x=y)=(x>y)+(x=y) \\
& =(x \geq y)-(x \leq y) \otimes(x \geq y)+(x \leq y) \otimes(x \geq y) .
\end{aligned}
$$

Complements of fuzzy partial order relations are defined by the following expressions:

$$
\begin{aligned}
\neg(x \leq y)= & (x<>y) \vee(x>y)=(x<>y)+(x>y) \\
= & 1-(x \geq y)-(x \leq y)+(x \leq y) \otimes(x \geq y) \\
& +(x \geq y)-(x \leq y) \otimes(x \geq y)=1-(x \leq y),
\end{aligned}
$$




$$
\begin{aligned}
\neg(x \geq y)= & (x<>y) \vee(x<y)=(x<>y)+(x<y) \\
= & 1-(x \geq y)-(x \leq y)+(x \leq y) \otimes(x \geq y) \\
& +(x \leq y)-(x \leq y) \otimes(x \geq y)=1-(x \geq y) .
\end{aligned}
$$

Another reflexive, symmetric and transitive fuzzy similarity relation is defined by the following expression:

$$
\begin{aligned}
(x \Longleftrightarrow y)= & (x=y) \vee(x<>y)=(x=y)+(x<>y) \\
= & (x \leq y) \otimes(x \geq y)+1-(x \geq y)-(x \leq y) \\
& +(x \leq y) \otimes(x \geq y) \\
= & 1-(x \geq y)-(x \leq y)+2(x \leq y) \otimes(x \geq y) .
\end{aligned}
$$

The complement of this relation is the fuzzy dissimilarity relation, defined by the following expression:

$$
\begin{aligned}
(x \underline{\vee} y)= & \neg(x \Longleftrightarrow y)=(x<y) \vee(x>y) \\
= & (x<y)+(x>y) \\
= & (x \leq y)-(x \leq y) \otimes(x \geq y)+(x \geq y) \\
& -(x \leq y) \otimes(x \geq y) \\
= & (x \leq y)+(x \geq y)-2(x \leq y) \otimes(x \geq y) .
\end{aligned}
$$

Complements of fuzzy strict order relations are defined by the following expression:

$$
\begin{aligned}
\neg(x<y)= & (x<>y) \vee(x>y) \vee(x=y) \\
= & (x<>y)+(x>y)+(x=y) \\
= & 1-(x \geq y)-(x \leq y)+(x \leq y) \otimes(x \geq y) \\
& +(x \geq y)-(x \leq y) \otimes(x \geq y) \\
& +(x \leq y) \otimes(x \geq y) \\
= & 1-(x \leq y)+(x \leq y) \otimes(x \geq y)=1-(x<y), \\
\neg(x>y)= & (x<>y) \vee(x<y) \vee(x=y) \\
= & (x<>y)+(x<y)+(x=y) \\
= & 1-(x \geq y)-(x \leq y)+(x \leq y) \otimes(x \geq y) \\
& +(x \leq y)-(x \leq y) \otimes(x \geq y) \\
& +(x \leq y) \otimes(x \geq y) \\
= & 1-(x \geq y)+(x \leq y) \otimes(x \geq y)=1-(x>y) .
\end{aligned}
$$

The complement of a fuzzy incomparability relation (relatedness or comparability relation) is defined by the following expression:

$$
\begin{aligned}
\neg(x<>y)= & (x<y) \vee(x>y) \vee(x=y) \\
= & (x<y)+(x>y)+(x=y) \\
= & (x \leq y)-(x \leq y) \otimes(x \geq y)+(x \geq y) \\
& -(x \leq y) \otimes(x \geq y)+(x \leq y) \otimes(x \geq y) \\
= & (x \leq y)+(x \geq y)-(x \leq y) \otimes(x \geq y) \\
= & 1-(x<>y) .
\end{aligned}
$$

Another fuzzy dissimilarity relation, complement of equivalence relation given in (29), is defined by the following expression:

$$
\begin{aligned}
(x \neq y)= & \neg(x=y)=(x<y) \vee(x>y) \vee(x<>y) \\
= & (x<y)+(x>y)+(x<>y) \\
= & (x \leq y)-(x \leq y) \otimes(x \geq y)+(x \geq y) \\
& -(x \leq y) \otimes(x \geq y)+1-(x \geq y)-(x \leq y) \\
& +(x \leq y) \otimes(x \geq y) \\
= & 1-(x \leq y) \otimes(x \geq y)=1-(x=y) .
\end{aligned}
$$

On the basis of information provided by the Boolean algebra of fuzzy relations, objects can be easily categorized in the artificial process of cognition. For example, the categorization process in which objects are recognized on the basis of their resemblance to prototypes can be formalized by using any of aforementioned similarity (or dissimilarity) relations. This is demonstrated in the following section.

\section{Illustrative Example}

In this section, the simple artificial cognitive system which mimics human categorization activity is constructed. The system categorizes objects on the basis of their similarities to prototypes of relevant categories. This will be demonstrated by using iris flower data set taken from [28]. The data set contains information on 150 four-dimensional objects, which belong to one of the three categories: $I_{\mathrm{Se}}, I_{\mathrm{Ve}}$, and $I_{\mathrm{Vi}}$-Iris setosa, Iris versicolor and Iris virginica, respectively. There are 50 objects in each category. Such objects can be defined by the following expression:

$$
o=[s l(o), s w(o), p l(o), p w(o)] .
$$

Here $o \in R^{4}$ is a physical object; $s l(o), s w(o), p l(o)$, and $p w(o)$ are the physical properties of object $o$-sepal length, sepal width, petal length, and petal width, respectively. 
The artificial cognitive system maps the physical object $o \in O$ into "mental object" $x$ by using the following expression:

$$
\begin{aligned}
x= & {\left[\frac{s l(o)}{\max _{o_{i} \in O}\left(s l\left(o_{i}\right)\right)}, \frac{s w(o)}{\max _{o_{i} \in O}\left(s w\left(o_{i}\right)\right)},\right.} \\
& \left.\frac{p l(o)}{\max _{o_{i} \in O}\left(p l\left(o_{i}\right)\right)}, \frac{p w(o)}{\max _{o_{i} \in O}\left(p w\left(o_{i}\right)\right)}\right] \\
= & {[S L(x), S W(x), P L(x), P W(x)] . }
\end{aligned}
$$

Here $x \in[0,1]^{4}$ is an object considered in the process of cognition, $S L(x), S W(x), P L(x), P W(x) \in[0,1]$ are the properties of object $x$, and $O$ is the set of objects or the domain of cognition.

Information on category membership is provided to the artificial cognitive system for 105 randomly selected objects. Set $X$ is the set of the remaining 45 objects (for categorization); set $Y$ is the set of prototypes for the three relevant categories. The task of the system is to categorize objects, based on their similarities to prototypes. The system builds prototypes on the basis of observed category members, that is, by using 105 randomly selected objects. For illustration purposes, prototypes are defined in two ways. In the first case, the prototype of category $C$ is the object $y_{C} \in Y$, whose properties are the averages of properties of all category members:

$$
\begin{aligned}
y_{C} & =\frac{1}{|C|} \sum_{z \in C} z \\
& =\left[\frac{1}{|C|} \sum_{z \in C} S L(z), \frac{1}{|C|} \sum_{z \in C} S W(z), \frac{1}{|C|} \sum_{z \in C} P L(z),\right. \\
& \left.\quad \frac{1}{|C|} \sum_{z \in C} P W(z)\right] \\
& =\left[S L\left(y_{C}\right), S W\left(y_{C}\right), P L\left(y_{C}\right), P W\left(y_{C}\right)\right] .
\end{aligned}
$$

Here $y_{C} \in[0,1]^{4}$ is the prototype of category $C, z \in[0,1]^{4}$ is the member of $C$, and $|C|$ is the cardinality of $C$.

In the second case, all observed members of a category are used as the prototype of that category. This is the exemplar approach. In both approaches, similarity between object $x \in$ $X$ and prototype (or exemplar) $y_{C} \in Y$ of category $C$ is defined by the following expression:

$$
\begin{aligned}
\left(x \Longleftrightarrow y_{C}\right)= & 1-\left(x \leq y_{C}\right)-\left(x \geq y_{C}\right) \\
& +2\left(x \leq y_{C}\right) *\left(x \geq y_{C}\right) .
\end{aligned}
$$

Here $\left(x \Leftrightarrow y_{C}\right) \in[0,1]$ is the Boolean consistent fuzzy similarity relation defined in $(34) ;\left(x \leq y_{C}\right) \in[0,1]$ and $\left(x \geq y_{C}\right) \in[0,1]$ are Boolean consistent fuzzy partial order relations defined in (28).
Boolean consistent fuzzy partial order relations are defined by the following expressions:

$$
\begin{aligned}
\left(x \leq y_{C}\right)= & \left(1-S L(x)+\min \left(S L(x), S L\left(y_{C}\right)\right)\right) \\
& *\left(1-S W(x)+\min \left(S W(x), S W\left(y_{C}\right)\right)\right) \\
& *\left(1-P L(x)+\min \left(P L(x), P L\left(y_{C}\right)\right)\right) \\
& *\left(1-P W(x)+\min \left(P W(x), P W\left(y_{C}\right)\right)\right), \\
\left(x \geq y_{C}\right)= & \left(x \leq y_{C}\right)^{-1} \\
= & \left(1-S L\left(y_{C}\right)+\min \left(S L\left(y_{C}\right), S L(x)\right)\right) \\
& *\left(1-S W\left(y_{C}\right)+\min \left(S W\left(y_{C}\right), S W(x)\right)\right) \\
& *\left(1-P L\left(y_{C}\right)+\min \left(P L\left(y_{C}\right), P L(x)\right)\right) \\
& *\left(1-P W\left(y_{C}\right)+\min \left(P W\left(y_{C}\right), P W(x)\right)\right) .
\end{aligned}
$$

In the first case, the artificial cognitive system assigns an object to a category whose prototype is the most similar to the object. This is defined by the following expression:

$$
C=\arg \max _{C \in\left\{I_{\mathrm{Se}}, I_{\mathrm{Ve}}, I_{\mathrm{Vi}}\right\}}\left(x \Longleftrightarrow y_{C}\right) \text {. }
$$

Here $x \in X, y_{C} \in Y$ is the prototype of category $C \in$ $\left\{I_{\mathrm{Se}}, I_{\mathrm{Ve}}, I_{\mathrm{Vi}}\right\}$, and $x, y_{\mathrm{C}} \in[0,1]^{4}$.

In the second case, an object is assigned to a category with the highest average similarity between the object and all category members (exemplars). This is defined by the following expression:

$$
C=\arg \max _{C \in\left\{I_{\mathrm{Se}}, I_{\mathrm{Ve}}, I_{\mathrm{Vi}}\right\}}\left(\frac{1}{|C|} \sum_{y_{\mathrm{C}} \in C}\left(x \Longleftrightarrow y_{C}\right)\right) .
$$

Here $y_{C} \in[0,1]^{4}$ is the exemplar of $C \in\left\{I_{\mathrm{Se}}, I_{\mathrm{Ve}}, I_{\mathrm{Vi}}\right\}$, and $|C|$ is the cardinality of category $C$.

The categorization process was executed 50 times. In each execution, 70 percent of randomly selected objects are used by the system for prototype formation. The remaining 30 percent of objects are then categorized. The accuracy of the categorization is measured as the average of the percentages of successfully categorized objects in each execution. In the first case, 95.56 percent of objects are successfully categorized. In the second case, 96.67 percent of objects are successfully categorized. As we can see, the artificial cognitive system based on the exemplar approach achieves slightly better results. The same results would have been obtained if the system had been constructed by using the Boolean consistent fuzzy dissimilarity relation defined in (35).

\section{Conclusion}

Categorization is a fundamental activity for any natural or artificial cognitive system. Formalization of this activity in accordance with the prototype theory of categorization is proposed. This is accomplished by using Boolean consistent 
fuzzy logic - the interpolative Boolean algebra. The proposed formalism is within the Boolean frame, securing the principles of categorization-perceived world structure and cognitive economy. Accordingly, an artificial cognitive system based on the proposed formalism can acquire maximum information about the world with the least computational effort. The universality of Boolean laws in the classical and the prototype theory shows that such laws are fundamental in the categorization process. The proposed artificial cognitive system achieves notable results in the considered example.

\section{Conflict of Interests}

The authors declare that there is no conflict of interests regarding the publication of this paper.

\section{References}

[1] H. Cohen and C. Lafebre, Handbook of Categorization in Cognitive Science, Elsevier, New York, NY, USA, 2005.

[2] J. S. Bruner, J. J. Goodnow, and G. A. Austin, A Study of Thinking, John Wiley \& Sons, New York, NY, USA, 1956.

[3] G. P. Goodwin and P. N. Johnson-Laird, "Mental models of Boolean concepts," Cognitive Psychology, vol. 63, no. 1, pp. 3459,2011

[4] J. Feldman, "A catalog of Boolean concepts," Journal of Mathematical Psychology, vol. 47, no. 1, pp. 75-89, 2003.

[5] E. Rosch and B. B. Lloyd, "Principles of categorization," in Cognition and Categorization, pp. 27-48, Lawrence Erlbaum Associates, Hillsdale, NJ, USA, 1978.

[6] J. A. Hampton, "Concepts as prototypes," Psychology of Learning and Motivation: Advances in Research and Theory, vol. 46, pp. 79-113, 2006.

[7] R. M. Nosofsky and S. R. Zaki, "Exemplar and prototype models revisited: response strategies, selective attention, and stimulus generalization," Journal of Experimental Psychology: Learning Memory and Cognition, vol. 28, no. 5, pp. 924-940, 2002.

[8] E. Rosch, "Slow lettuce: categories, concepts, fuzzy sets, and logical deduction," in Concepts and Fuzzy Logic, pp. 89-120, The MIT Press, 2011.

[9] L. A. Zadeh, "Fuzzy sets," Information and Computation, vol. 8, no. 3, pp. 338-352, 1965.

[10] L. A. Zadeh, "A note on prototype theory and fuzzy sets," Cognition, vol. 12, no. 3, pp. 291-297, 1982.

[11] D. G. Radojevic, "Fuzzy set theory in the Boolean frame," International Journal of Computers Communications \& Control, vol. 3, no. 3, pp. 121-131, 2008.

[12] D. G. Radojević, "New [0,1]-valued logic: a natural generalization of Boolean logic," Yugoslav Journal of Operations Research, vol. 10, no. 2, pp. 185-216, 2000.

[13] L. A. Zadeh, "Outline of a new approach to the analysis of complex systems and decision processes," IEEE Transactions on Systems, Man, and Cybernetics, vol. 3, pp. 28-44, 1973.

[14] D. Radojević, "Real-valued realizations of boolean algebras are a natural frame for consistent fuzzy logic," Studies in Fuzziness and Soft Computing, vol. 299, pp. 559-565, 2013.

[15] L. A. Zadeh, "Is there a need for fuzzy logic?" Information Sciences, vol. 178, no. 13, pp. 2751-2779, 2008.

[16] L. A. Zadeh, "From computing with numbers to computing with words-from manipulation of measurements to manipulation of perceptions," IEEE Transactions on Circuits and Systems. I. Fundamental Theory and Applications, vol. 46, no. 1, pp. 105-119, 1999.

[17] L. A. Zadeh, "A new direction in AI-toward a computational theory of perceptions," AI Magazine, vol. 22, no. 1, pp. 73-84, 2001.

[18] J. Fodor and M. Roubens, Fuzzy Preference Modelling and Multicriteria Decision Support, Kluwer Academic Publishers, Dordrecht, The Netherlands, 1994.

[19] D. N. Osherson and E. E. Smith, "On the adequacy of prototype theory as a theory of concepts," Cognition, vol. 9, no. 1, pp. 35$58,1981$.

[20] H. Kamp and B. Partee, "Prototype theory and compositionality," Cognition, vol. 57, no. 2, pp. 129-191, 1995.

[21] P. N. Johnson-Laird, Mental Models: Towards a Cognitive Science of Language, Inference, and Consciousness, Harvard University Press, Cambridge, Mass, USA, 1983.

[22] D. Radojević, "Real sets as consistent Boolean generalization of classical sets," in From Natural Language to Soft Computing: New Paradigms in Artificial Intelligence, pp. 150-171, Editing House of Romanian Academy, Bucharest, Romania, 2008.

[23] P. Milošević, B. Petrović, D. Radojević, and D. Kovačević, "A software tool for uncertainty modeling using Interpolative Boolean algebra," Knowledge-Based Systems, vol. 62, pp. 1-10, 2014.

[24] R. M. Nosofsky, "Exemplar-based approach to relating categorization, identification, and recognition," in Multidimensional Models of Perception and Cognition, pp. 363-393, Lawrence Erlbaum Associates, New York, NY, USA, 1992.

[25] D. L. Medin and M. M. Schaffer, "Context theory of classification learning," Psychological Review, vol. 85, no. 3, pp. 207-238, 1978.

[26] D. Radojević, "Logical aggregation based on interpolative boolean algebra," Mathware \& Soft Computing, vol. 15, no. 1, pp. 125-141, 2008.

[27] D. Radojević, "Interpolative relations and interpolative preference structures," Yugoslav Journal of Operations Research, vol. 15, no. 2, pp. 171-189, 2005.

[28] K. Bache and M. Lichman, UCI Machine Learning Repository, 2013, http://archive.ics.uci.edu/ml. 


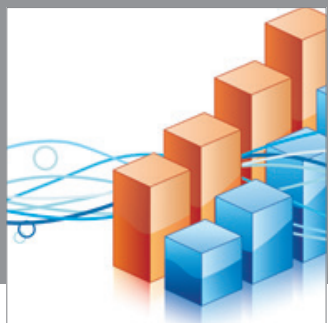

Advances in

Operations Research

mansans

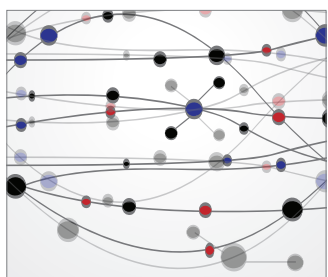

The Scientific World Journal
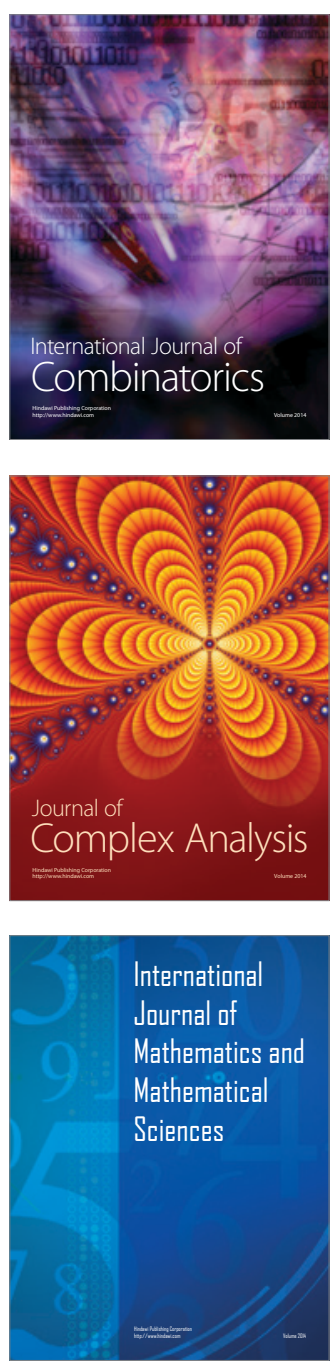
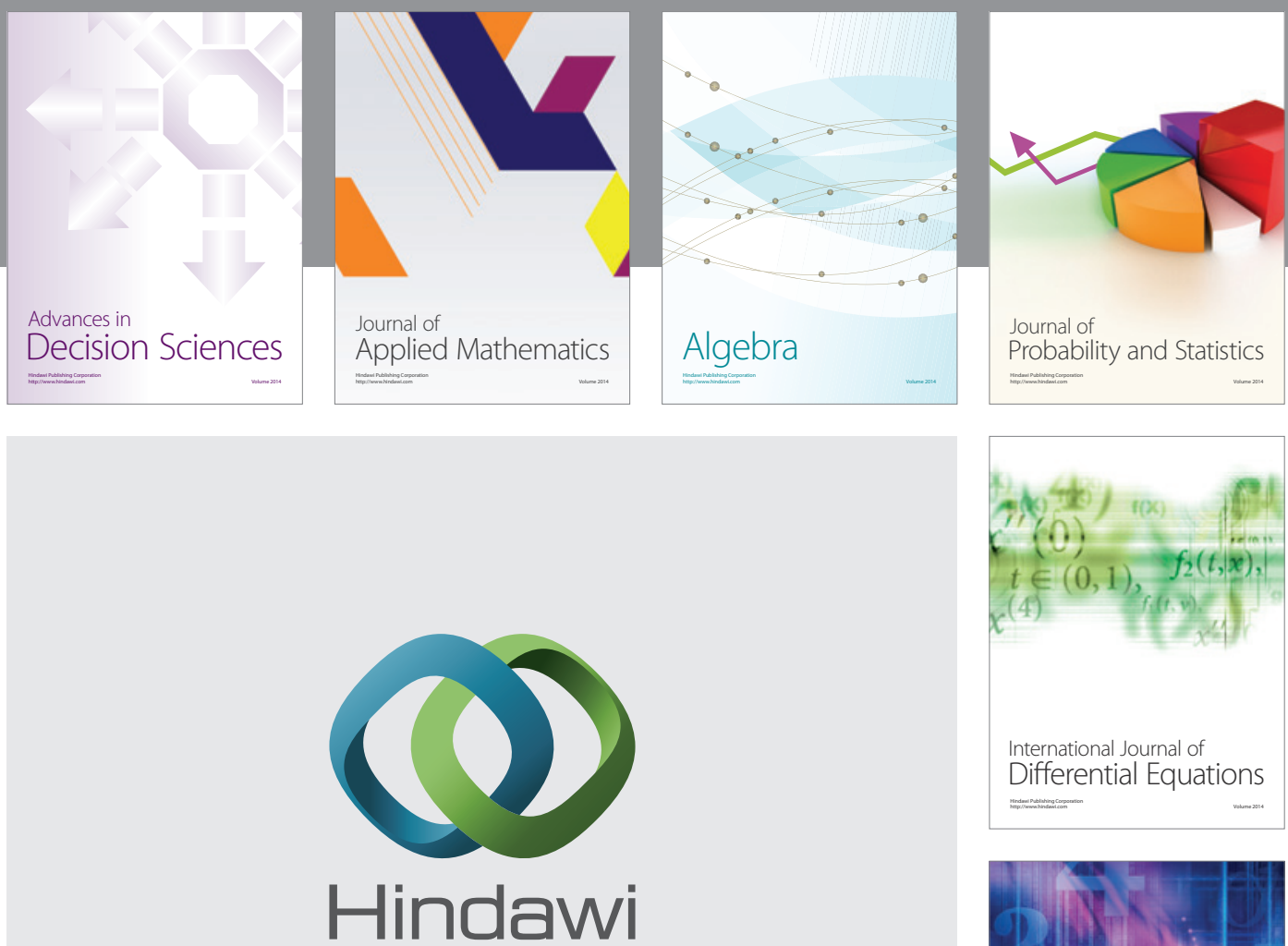

Submit your manuscripts at http://www.hindawi.com
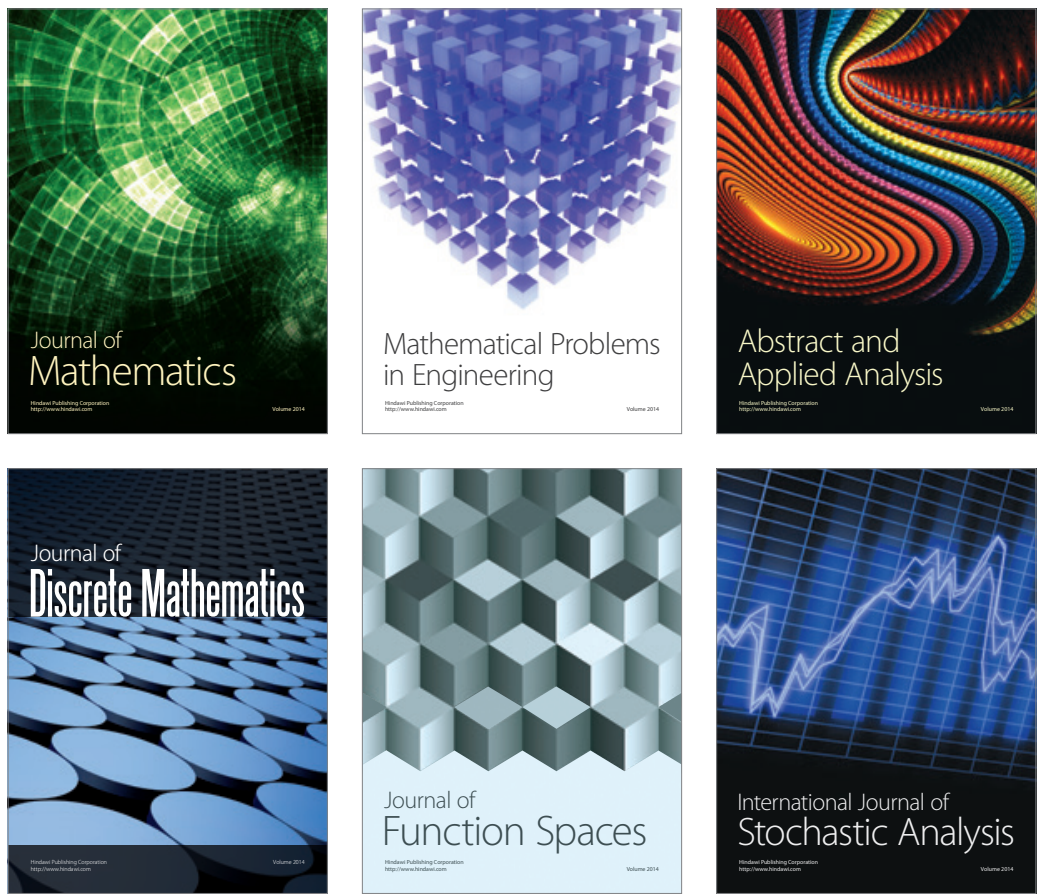

Journal of

Function Spaces

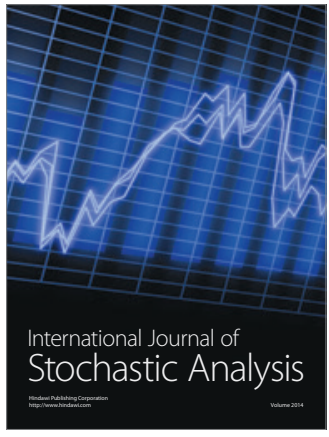

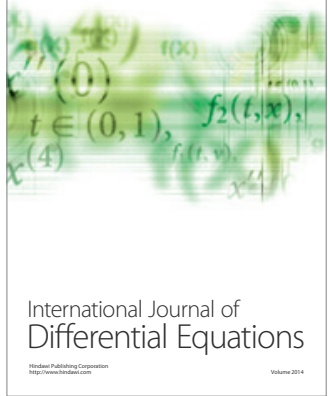
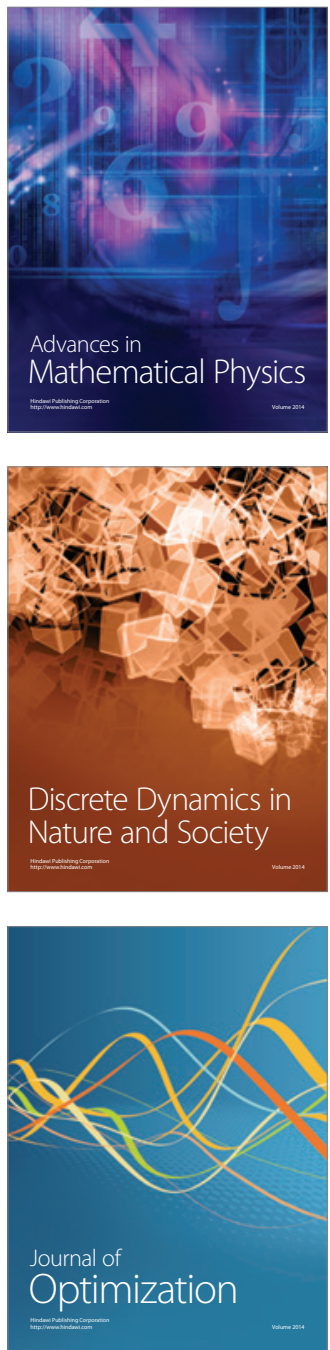